\title{
Elastic interaction energy between a silicon interstitial and a carbon substitutional in a silicon crystal
}

\author{
Ursula M. E. Christmas, David A. Faux, * and Nicholas E. B. Cowern \\ Advanced Technology Institute, University of Surrey, Guildford, GU2 4ES, United Kingdom \\ (Received 8 March 2007; revised manuscript received 23 July 2007; published 13 November 2007)
}

\begin{abstract}
The strain interaction energy between a silicon interstitial and a carbon substitutional in a silicon crystal was modeled by a continuum Green's function method and by atomistic simulation. The interaction energy is proportional to $d^{-3}$, where $d$ is separation distance between the defects. The pair interaction energy was found to be less than $0.04 \mathrm{meV}$ for $d>6 \mathrm{~nm}$ increasing to more than about $0.1 \mathrm{meV}$ for $d<3 \mathrm{~nm}$. The energies are unlikely to influence the diffusional behavior of the defects except at distances of one or two unit cells. The potential between the point defects is repulsive if they are oriented along the (100) crystal axis, but attractive if they are positioned along (110) or (111).
\end{abstract}

DOI: 10.1103/PhysRevB.76.205205

PACS number(s): 61.72.Ji, 61.72.-y, 62.20.Fe

\section{INTRODUCTION}

Silicon, one of the most widely used materials in the electronics industry, often contains carbon to inhibit the diffusion of dopants such as boron because the outdiffusion of boron from as-grown regions can significantly degrade device performance. ${ }^{1}$ But carbon itself diffuses surprisingly fast. This study examines whether the strain interaction energy between point defects contributes to the fast diffusion of carbon in silicon crystals.

Implantation of boron in silicon by ion beam causes an excess of silicon self-interstitials. ${ }^{2}$ Ion implantation also introduces defects into the crystal which are repaired by annealing at a temperature of about $800^{\circ} \mathrm{C}$. Boron diffuses by kick-in and kick-out reactions with silicon interstitials, ${ }^{3,4}$ as illustrated in Fig. 1 and diffuses particularly fast during annealing. ${ }^{2}$ Diffusion of dopants is undesirable; the dopant profile needs to be kept sharply steplike and this becomes more important as device sizes reduce. Carbon is therefore added to provide a sink for the self-interstitials, inhibiting the diffusion of boron. ${ }^{2,5,6}$ But carbon itself diffuses fast; it is even more diffusive in silicon than boron. ${ }^{7}$ Like boron, carbon diffuses by kick-out and kick-in reactions with silicon interstitials ${ }^{8}$ in which a silicon interstitial kicks a substitutional carbon atom out from a lattice site moving the carbon atom into an interstitial site. The carbon interstitial then "kicks in," replacing a silicon atom on a lattice site, as illustrated in Fig. 1.

Models in which the carbon substitutional and the silicon interstitial meet each other by chance do not account for the observed fast rate of diffusion of carbon in silicon, unless a large value is attributed to the capture radius (a fitting parameter representing the separation distance within which a reaction is inevitable ${ }^{9}$ ). One contributory factor might be the strain fields associated with a carbon substitutional and a silicon interstitial interact, which produce an attractive force between the two defects. The interaction energy may produce a "potential well" that draws two defects towards each other increasing the effective capture radius.

In this paper, we estimate the strain interaction energy between a silicon self-interstitial and a carbon substitutional using two different approaches. In the first method, which is akin to a local force model, the defects were modeled as cubic inclusions in a silicon continuum using a Green's function method to estimate the interaction energy due to the strain fields. Secondly, we used atomistic simulation as an approximation in which the silicon interstitial was represented by a larger substitutional and the carbon by a smaller substitutional in a lattice of silicon atoms. These two approaches were chosen to provide estimates of the defect interaction energy over complementary length scales, the continuum Green's function approach valid for larger distances, and the atomistic simulations for defect pair separations of a few unit cells. This work is complementary to that of Hobler and $\mathrm{Kresse}^{10}$ who presented results for interstitial and vacancy pair defects in silicon using $a b$ initio for defect pairs separated by up to two unit cells.

The first of the two models, the Green's function approach, is based on the local force model, which is one of the simplest models of a point defect in continuum elasticity. A pair of opposing forces is placed along each axis ${ }^{11}$ around the defect, equivalent to inserting a spherical inclusion into a spherical hole of initially different size. ${ }^{12}$ Green's functions and Radon ${ }^{11}$ or Fourier ${ }^{12,13}$ transforms can then be used to calculate strain and interaction energy. A refinement of this model, more suitable for the near field, is the "nonlocal" continuum model, which places an array of radial forces at the positions of the defect's first and second neighbor atoms. ${ }^{14}$ The forces used in this model are derived from the harmonic lattice model developed by Bullough and Hardy. ${ }^{15}$ All of these models are capable of describing an anisotropic material such as silicon. At a distance $d$ of 10 unit cells from the defect, the displacement predicted by the local force

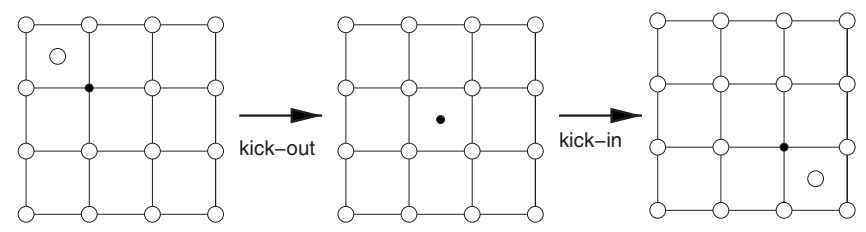

FIG. 1. Two-dimensional representation of kick-in and kick-out reactions between a substitutional and a self-interstitial. The black dot represents a boron or carbon atom in a silicon lattice. 
model differs from that of the nonlocal force model by $\approx 10 \%{ }^{14}$ and as $d \rightarrow \infty$ the two values become equal. In the local force model the elastic interaction energy between two defects is proportional to $d^{-3}$ while the nonlocal force and harmonic lattice models predict a $d^{-5}$ relation. ${ }^{12,16,17}$ The harmonic lattice model predicts that, for $\mathrm{Cu}$, the interaction energy between two like point defects is negative when they are both lying on the (100) crystal axis and positive in the (110) and (111) orientations. The Green's function model described in the following section produces a similar conclusion for silicon (with signs reversed for the two unlike defects).

The second approach to the estimation of the interaction energy of a carbon substitutional and a silicon self-interstitial is based on a simple atomistic model. Standard classical interatomic potentials are used and a molecular dynamics package is used to calculate the system energy at $0 \mathrm{~K}$. This approach has been used, for example, to estimate the strain energies in semiconductor materials. ${ }^{18}$

This paper is organized as follows. In Sec. II we derive the expression for strain interaction energy and describe both the Green's functions and atomistic methods. Results are presented in Sec. III. Despite the substantial differences in approach and the approximations used, both methods are shown to draw quantitatively similar results. Finally, the conclusions are presented in Sec. IV.

\section{THEORY}

\section{A. Green's function method}

Strain energy $w$ per unit volume is defined as

$$
w=\frac{1}{2} \sigma_{i j} \varepsilon_{i j}=\frac{1}{2} C_{i j k l} \varepsilon_{k l} \varepsilon_{i j},
$$

where $\sigma, \epsilon$, and $C$ are the stress, strain, and stiffness tensors, the subscripts represent the three dimensions in space, and the sum is taken over all values of repeated indices. Total energy $W$ in a volume $\mathrm{V}$ is given by

$$
W=\int \frac{1}{2} C_{i j k l} \varepsilon_{k l} \varepsilon_{i j} d V
$$

Assuming stresses and strains add linearly, the strain energy density due to two point defects is

$$
\begin{aligned}
w^{(1+2)} & =\frac{1}{2} C_{i j k l}\left(\varepsilon_{i j}^{(1)}+\varepsilon_{i j}^{(2)}\right)\left(\varepsilon_{k l}^{(1)}+\varepsilon_{k l}^{(2)}\right) \\
& =\frac{1}{2} C_{i j k l}\left(\varepsilon_{i j}^{(1)} \varepsilon_{k l}^{(1)}+\varepsilon_{i j}^{(2)} \varepsilon_{k l}^{(2)}+\varepsilon_{i j}^{(1)} \varepsilon_{k l}^{(2)}+\varepsilon_{i j}^{(2)} \varepsilon_{k l}^{(1)}\right) .
\end{aligned}
$$

The first two terms are the energy densities associated with single defects and are not affected by the presence of the second defect. The last two terms are the energy densities $\Delta w$ associated with the interaction between the two defects. The interaction energy $\Delta W$ is therefore given by

$$
\Delta W=\frac{1}{2} \int C_{i j k l}\left(\varepsilon_{k l}^{(1)} \varepsilon_{i j}^{(2)}+\varepsilon_{k l}^{(2)} \varepsilon_{i j}^{(1)}\right) d V=\int C_{i j k l} \varepsilon_{i j}^{(1)} \varepsilon_{k l}^{(2)} d V,
$$

since $C_{i j k l}=C_{k l i j}$.

In the Green's function model, at the site of the silicon interstitial, a silicon unit cell is removed and replaced by a larger cube, which is initially compressed to fit into the unit cell. The initial strain inside the cube, before the system is allowed to relax, is known as misfit strain, and is uniform throughout the cell and zero elsewhere. When the system relaxes, the cube expands and a nonuniform strain arises inside and outside the cell. The carbon substitutional is represented by a corresponding smaller cube replacing a unit cell of silicon; thus the misfit strain has the opposite sign to that of the interstitial cell. The use of a cube is arbitrary, but the strain field a distance of just one unit cell away from the source of the strain is almost independent of the shape of the source. ${ }^{19}$

It is now necessary to estimate the effective misfit strain of a cube of silicon, which contains an extra atom in the form of an interstitial. Misfit strain $\varepsilon^{*}$ for a small volume of material $A$ embedded in material $B$ is defined as

$$
\varepsilon^{*}=\frac{a_{B}-a_{A}}{a_{A}},
$$

where $a_{A}$ and $a_{B}$ are the lattice constants of $A$ and $B$. The lattice constant of silicon is $0.543 \mathrm{~nm}$. The cell which includes the silicon interstitial contains nine atoms instead of the usual eight. The unstrained volume of nine silicon atoms is therefore $9 / 8$ of a normal silicon unit cell. To find a characteristic length, comparable with $a$, we take the cube root of the volume. Hence the representative misfit strain of the silicon cell containing an interstitial is given by

$$
\varepsilon_{\mathrm{Sil}}^{*}=\frac{1-\left(\frac{9}{8}\right)^{1 / 3}}{\left(\frac{9}{8}\right)^{1 / 3}} .
$$

The cell representing the carbon substitutional contains seven silicon atoms and one carbon atom, so the total unstrained volume is taken to be $\frac{7}{8} a_{S i}^{3}+\frac{1}{8} a_{C}^{3}$, and the misfit strain is given by

$$
\varepsilon_{\mathrm{C}}^{*}=\frac{a_{\mathrm{Si}}-\left(\frac{7}{8} a_{\mathrm{Si}}^{3}+\frac{1}{8} a_{\mathrm{C}}^{3}\right)^{1 / 3}}{\left(\frac{7}{8} a_{\mathrm{Si}}^{3}+\frac{1}{8} a_{\mathrm{C}}^{3}\right)^{1 / 3}} .
$$

A Green's function method is used to deduce the relaxation strains. A Green's function is the response (in this case the strain response) at a field point (anywhere in the crystal) due to a unit impulse at a source point (inside one of the cells containing a point defect). Integrating over the volume where impulses occur (in this case, the defect cell) gives the total response. We used the Green's tensors developed by Faux et al. $^{20,21}$ for materials with cubic anisotropy based on the work of Mura and Kinoshita. ${ }^{22}$ Faux's method is particularly suitable for this computationally intensive problem as the Green's functions form an infinite polynomial series, where the first term describes the isotropic case and subsequent terms are successively higher-order corrections for aniso- 

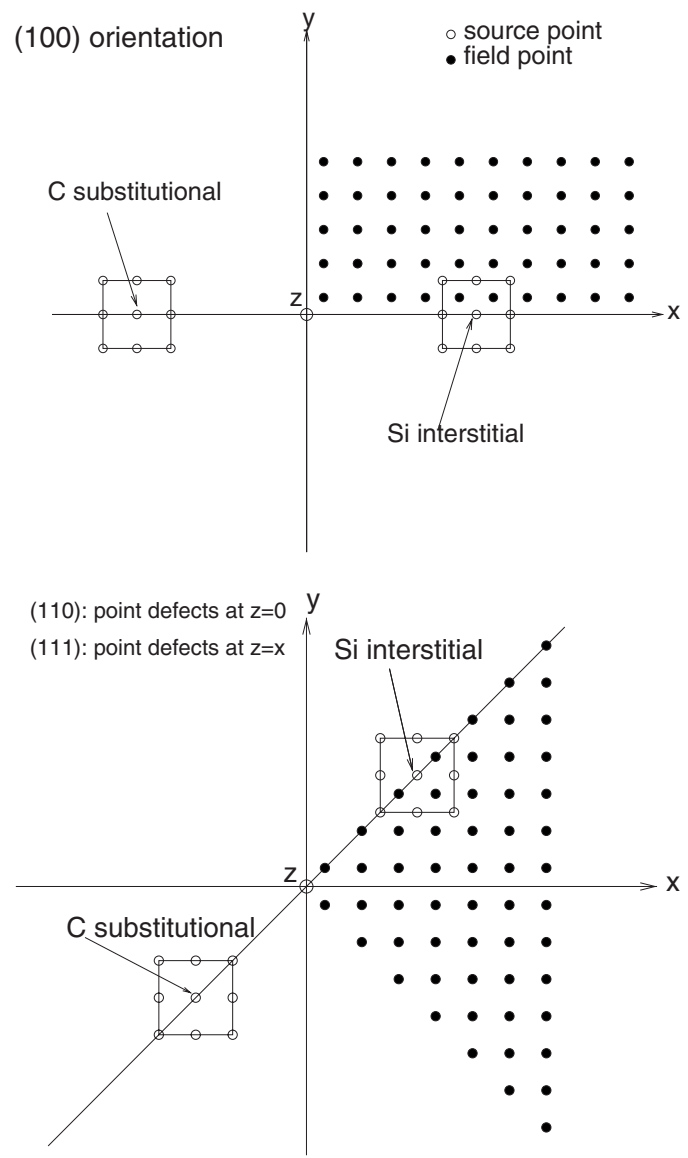

FIG. 2. Schematic diagram showing the geometry in cross section: (a) the (100) orientation and (b) the (110) orientation, where field points at $z>0$ in the quadrant indicated are integrated over, and the (111) orientation, where integration occurs over both positive and negative $z$ values.

tropy, and they are therefore fast to compute. Faux has shown that truncation after the third term produces an excellent approximation to the exact solution. ${ }^{20}$ The more general and more complex method of $\operatorname{Pan}^{23,24}$ takes into account the piezoelectric effect (zero for silicon) and can be expressed as a surface integral, which is useful for dealing with the numerical singularities that arise when integrating through the source volume.

The calculation involved two nested sets of numerical integrals; first, integration is required over source points to calculate the strain at a field point due to each of the two point defects then, second, the product of these strains is integrated over field points in a large volume of material surrounding the point defects in order to calculate the total strain interaction energy [Eq. (4)]. Three different defect orientations were modeled with defects orientated along the (100), (110), and the (111) crystal axes, respectively. The geometry of our model is shown in Fig. 2. For symmetry reasons it was only necessary to take field points in $1 / 8$ of the total volume in the (100) and (110) cases [1/4 of the total volume in the (111) case].

In the case of the inner integral, a Simpson weighting was used to increase the accuracy. For field points close to the defects, the mesh was refined by a factor of 3 . The disadvantage of integrating over a source volume as opposed to a surface integral over the source surface is that the interaction energy is highly sensitive to the distance from a field point to the closest source point. So, for field points inside the defect cell, we assumed a uniform value for the strain throughout the cell. This value of the constant strain used in this region was based on the strain at the center of the cell as calculated by the Pan method ${ }^{23}$ (which uses a surface integral). This approximation introduces an error of less than $8 \%$ in the total strain interaction energy.

The field points extend 200 cells beyond the source points in each direction. By a semi-analytical integration out to infinity, we have determined that this accounts for more than $99.96 \%$ of the total strain interaction energy of an infinitely large silicon crystal containing the two defects, since the strain decays as $r^{-3}$, where $r$ is the distance between the source point and the field point. For field points far from the source points, the density of field points was reduced to save computational time.

We used the following material parameters for silicon: elastic stiffness $C_{11}=166 \mathrm{GPa}, C_{12} \quad$ (Lame constant $\lambda$ ) $=64 \mathrm{GPa}, C_{44}($ Lame constant $\mu)=79.6 \mathrm{GPa}$, lattice constant $a=0.543 \mathrm{~nm}$. For carbon we used the lattice constant of diamond $(0.356 \mathrm{~nm})$. In each of the three orientations we took a range of distances $d$ between point defects. The model is probably only valid for $d>\sim 10$ unit cells or $5 \mathrm{~nm}$.

\section{B. Atomistic method}

Atomistic calculations were undertaken, first, to check the order of magnitude of the strain interaction energies obtained by continuum calculations as described in the previous section and, second, as a means of obtaining reasonable estimates of the strain interaction energies at shorter distances.

We adopt a simplistic model in which a silicon crystal has two atoms replaced, one by a siliconlike atom with a larger size (to represent the interstitial) and one replaced by a smaller siliconlike atom (to approximate the carbon interstitial). This model has obvious shortcomings and would not be expected to accurately simulate the real defect pair especially at very short distances but should serve to check the reasonableness of Green's function calculation, recognizing that the Green's function calculation is itself subject to approximations, and extend the range of the energy calculation to somewhat shorter distances. The key approximations inherent in the atomistic model is the size of the Si-like atoms to best approximate the interstitial silicon and substitutional carbon. We acknowledge, also, that there are more sophisticated interatomic potentials than the Stillinger-Weber/Jian set used here including potentials which explicitly treat silicon-carbon interactions which are likely to produce a more accurate numerical result for the interaction energy. It is also noted that a major contribution to the energy is due to the strain of the many atoms surrounding the defects and the accuracy of the various interatomic potentials at high distortions is not known.

The atomistic computer simulations are performed on a cube of silicon with dimensions of $23 \times 0.543095 \mathrm{~nm}$ con- 
taining a total of 97336 atoms. Stillinger-Weber ${ }^{26}$ (SW) interatomic potentials are used with the modification proposed by Jian $e t a l .{ }^{25}$ The Jian $e t$ al. potential parameters were used because the bulk modulus agrees with experiment whereas the original SW parameterization yields a value about $6 \%$ too large. The Stillinger-Weber potentials contain a two-body component of the form

$$
\phi_{i j}^{(2)}=\varepsilon A\left(B r^{-4}-1\right) \exp \left\{\delta(r-b)^{-1}\right\}
$$

where $r=\left|\mathbf{r}_{i}-\mathbf{r}_{j}\right| / \sigma$ and $r<b\left(\phi_{i j}^{(2)}=0\right.$ when $\left.r \geq b\right)$. $\sigma$ is chosen so that this function has a minimum at $r=2^{1 / 6}$ with an energy $-\varepsilon$.

The three-body term is of the form

$$
\phi_{i j k}^{(3)}=\varepsilon \lambda\left(\cos \theta+\frac{1}{3}\right)^{2} \exp \left\{\gamma\left(r_{i j}-b\right)^{-1}+\gamma\left(r_{i k}-b\right)^{-1}\right\},
$$

where $\theta$ is the angle between atom pairs $i j$ and $i k$. The dimensionless potential cutoff $b$ is 1.8 and $\gamma$ is 1.2 as chosen by Stillinger and Weber. ${ }^{26}$ The parameters $\varepsilon, A, B, \delta, \sigma_{\mathrm{SiSi}}$ and $\lambda$ are $2.17 \mathrm{eV}, 6.865464121,0.611445848,0.9757$, $2.0951 \AA$, and 9.11, respectively. ${ }^{25}$

The cube of silicon contains two model substitutional atoms, one larger $(L)$ than silicon and one smaller $(S)$, separated by a distance $d$ in either the (100) or (111) direction. The former approximates a silicon interstitial and the latter represents a carbon substitutional. The potentials defined above are used for atoms $L$ and $S$ with just the parameter $\sigma$ changing to reflect the different sizes of the substitutional defects. All other parameters associated with either the twobody or the three-body potential are unchanged. The atomistic simulations represent approximations to the real system and, therefore, it is difficult to assign appropriate values to $\sigma$ for the defect atoms. For example, Eqs. (6) and (7) produce values of $\approx 3 \%$ for the magnitude of the effective misfit strain for either the silicon interstitial or the carbon substitutional. The lattice constants for carbon and silicon suggest that a carbon substitutional should be associated with a $\sigma$ about 20\% smaller than that for silicon (assuming that the usual geometric combination is used for mixed atom pairs so, for example, $\left.\sigma_{\mathrm{SiL}}=\sqrt{\sigma_{\mathrm{SiSi}} \sigma_{L L}}\right)$. In practice, the potentials for the $\mathrm{Si}-L$ and $\mathrm{Si}-S$ interactions are assigned $\sigma_{\mathrm{Si} L}$ $=2.30461$ and $\sigma_{\mathrm{SiS}}=1.88559$, respectively, being chosen to be $10 \%$ larger and smaller than the value for $\mathrm{Si}$, respectively.

All simulations are performed using the DLPOLY ${ }^{27}$ molecular dynamics (MD) package using the NVT ensemble (constant number of atoms, volume, and temperature) at $0 \mathrm{~K}$ so the MD package is used simply to determine the system energy at the minimum energy configuration. Periodic boundary conditions are used. The system is equilibrated for about 30 ps using 0.005 ps time steps followed by 60 ps using $0.002 \mathrm{ps}$ time steps. The configurational energy is recorded to 12 significant figures. The computed temperature is typically $10^{-9}-10^{-10} \mathrm{~K}$ at the end of the run and the system is deemed to have equilibrated once the configurational energy has not changed for a minimum of $1 \mathrm{ps}$. It is likely that the true equilibrium energy has not been achieved, but the difference is judged to be, at most, 1 or 2 at the 12th signifi-

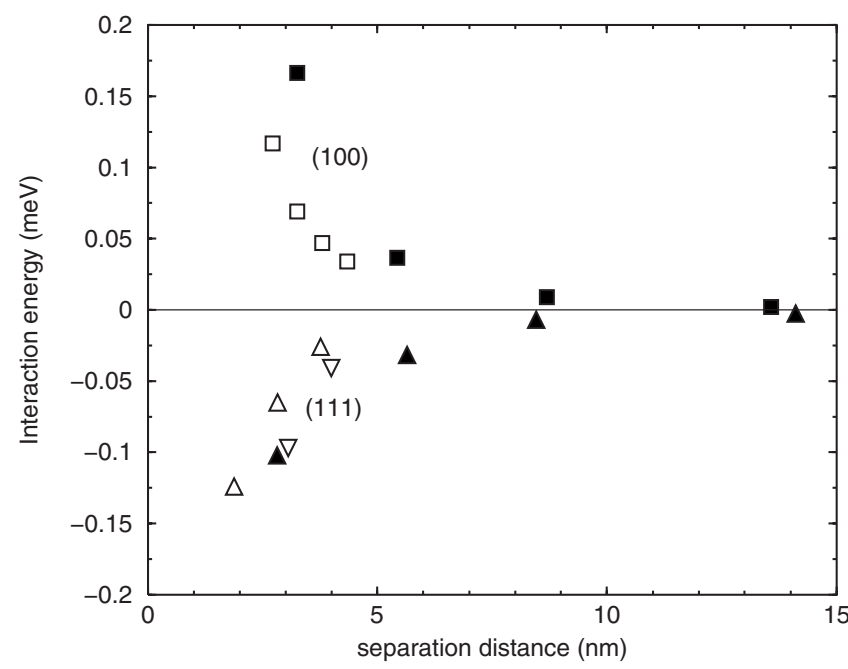

FIG. 3. The strain interaction energy between a silicon interstitial and a carbon substitutional point defect in a silicon crystal, as a function of separation distance between the defects. Squares denote the pair of defects lying along the (100) crystal axis and triangles along (111). Filled symbols are for the continuum Green's function method and unfilled for the atomistic method. $\nabla$ has the defects at the corner of the unit cell and $\triangle$ at $(1 / 4,1 / 4,1 / 4)$ in the unit cell.

cant figure equivalent to about $0.001-0.002 \mathrm{meV}$. Even though this systematic error is small, as a similar convergence criterion is used for all simulations, these systematic errors subtract out once the difference between energies is taken.

Simulations were run for atoms $S$ and $L$ separated by $1-7$ cell dimensions in the (100) direction and 1-4.25 cell dimensions in the (111) direction, a maximum separation of about $4 \mathrm{~nm}$.

\section{RESULTS AND DISCUSSION}

Figure 3 shows the interaction energy between a Si interstitial and a $\mathrm{C}$ substitutional separated by up to $15 \mathrm{~nm}$, calculated by the atomistic and the continuum Green's function methods. Figures 4 and 5 show the same data, but on a semilogarithmic plot extending out to a separation of $100 \mathrm{~nm}$.

The quantitative agreement between results from the atomistic and continuum models is impressive considering the various approximations used. Both indicate that strain interaction energy $\Delta W$ between a silicon interstitial and a carbon substitutional is less than about $0.04 \mathrm{meV}$, equivalent to a temperature of $5 \mathrm{~K}$, for separation distances greater than $5 \mathrm{~nm}$. This is small compared to $k T$ at room temperature $(25 \mathrm{meV})$ or higher. We can conclude that strain interaction potential does not play a significant role in attracting point defects to each other at distances greater than $5 \mathrm{~nm}$ except at low temperatures.

Both atomistic and continuum models agree that, for defects aligned along (100), the interaction energy $\Delta W$ is positive (so defects repel each other). They also agree that in the (111) orientation (at least at $d>2 \mathrm{~nm}$ ) $\Delta W$ is negative 


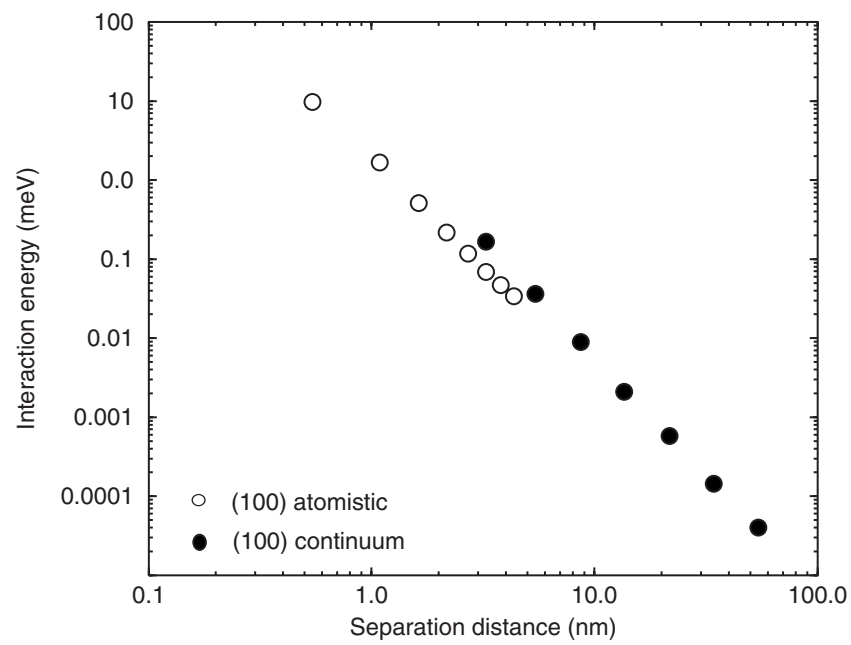

FIG. 4. Semilogarithmic plot of interaction energy for a pair of defects in the (100) orientation.

(which implies that defects attract each other). Our continuum model also predicts that the defects attract each other in the (110) orientation. This is consistent with the results of Masumura, ${ }^{13}$ Schaefer, ${ }^{28}$ Shneck, ${ }^{12}$ and others, all of whom use the local force model. All of these are studies of $\mathrm{Cu}$, which has cubic symmetry and the same sign of anisotropy coefficient $\left(\mathrm{C}_{11}-\mathrm{C}_{12}-2 \mathrm{C}_{44}\right)$ as $\mathrm{Si}$, so would be expected to behave in a similar way.

The difference in sign of energy between the various orientations can be explained as follows. The total interaction energy is dominated by the contributions from field points inside the defect cell, very close to the source. The element of strain energy density for a field point inside defect cell (1) is given by

$$
w=C_{i j k l} \epsilon_{i j}^{(1)} \epsilon_{k l}^{(2)}
$$

as in Eq. (4). At the center of defect cell (1), $\epsilon_{i j}^{(1)}$ is a scalar multiple $\epsilon^{(1)}$ of the identity matrix; let us assume it is positive, like that of the carbon substitutional. Thus the interaction energy $w_{c e n}$ at the center of source (1) is

$$
w_{c e n}=\left(\mathrm{C}_{11}+2 \mathrm{C}_{12}\right) \epsilon^{(1)} \epsilon_{i i}^{(2)}
$$

where $\epsilon_{i i}^{(2)}$ is the trace of the tensor for strain inside cell (1) due to defect (2). $\left(\mathrm{C}_{11}+2 \mathrm{C}_{12}\right)$ is always positive. The sign of $w_{c e n}$ (and thus of $\Delta W$ ) is therefore the same as that of $\operatorname{Tr}\left(\epsilon_{i j}^{(2)}\right)$. Assuming that defect (2) has negative misfit strain, like the silicon interstitial, $\operatorname{Tr}\left(\epsilon_{i j}^{(2)}\right)$ is positive when the two defects are oriented along (100), which is a soft direction, but negative along the hard directions (110) and (111). This observation accords with the arguments of Shneck. ${ }^{12}$ Of course, the curves would coincide for an isotropic medium.

The atomistic simulation found a large positive $\Delta W$ (about $2 \mathrm{meV}$, not shown on the graphs) for $d<\sim 1 \mathrm{~nm}$ in (111) orientation, in the configuration where the defects are at the corner of their unit cells. This agrees with the findings

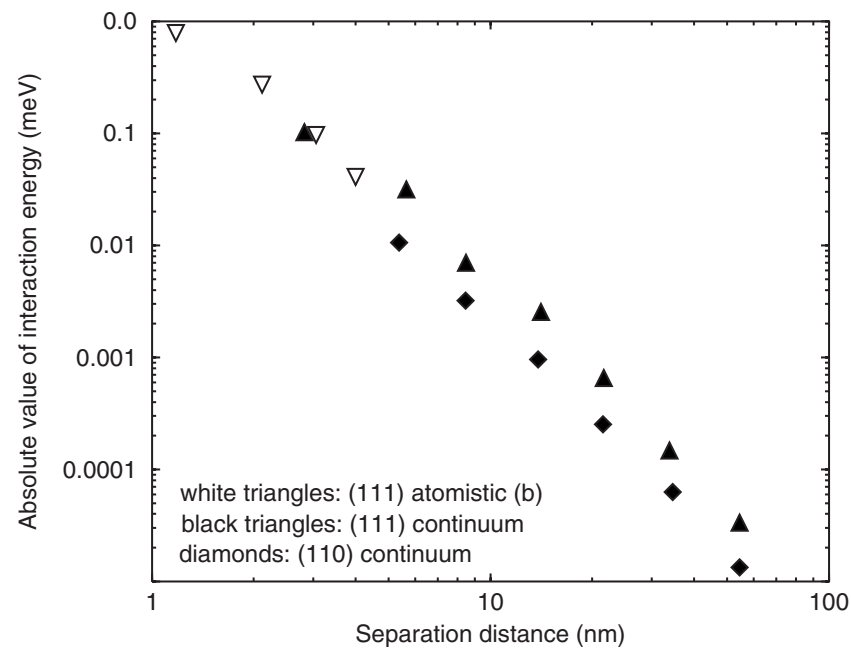

FIG. 5. Semilogarithmic plot of absolute value of interaction energy vs separation distance, for a pair of defects in the (110) and (111) orientations.

of Shneck. ${ }^{12}$ The continuum model would not be valid at such a small separation. This result suggests that the strain interaction energy may only contribute to the diffusion process once the two defects are close, less than about $3 \mathrm{~nm}$.

The straight lines on the semilogarithmic plots imply that strain interaction energy is proportional to $d^{-3}$, which agrees with the literature on the local force model. ${ }^{12}$ This follows from the fact that the strain Green's function for a particular orientation is proportional to $r^{-3}$, where $r$ is the distance from the source point to the field point.

\section{CONCLUSION}

We modeled two point defects in a Si crystal (a C substitutional and a $\mathrm{Si}$ interstitial) as inclusions in a continuous medium, and separately as large and small substitutionals in an atomistic simulation. The strain interaction energy $\Delta W$ between a $\mathrm{Si}$ interstitial and a $\mathrm{C}$ substitutional is found to be $<0.04 \mathrm{meV}$ for separation distance $d>5 \mathrm{~nm}$. This indicates that strain interaction energy plays a negligible part in the diffusion of $\mathrm{C}$ in $\mathrm{Si}$ at room temperature or above at distances greater than about $5 \mathrm{~nm}$. The strain interaction energy may contribute to the diffusion process at low temperatures and/or once the two defects are less than about $3 \mathrm{~nm}$ apart.

The potential between the defects due to the strain is repulsive if they are oriented along the (100) axis, but attractive along (110) and (111). $\Delta W$ is proportional to $d^{-3}$.

\section{ACKNOWLEDGMENT}

U.M.E. acknowledges the support of Engineering and Physical Sciences Research Council (UK). 
*d.faux@surrey.ac.uk

${ }^{1}$ S. Rizk, Y. M. Haddara, and A. Sibaja-Hernandez, J. Vac. Sci. Technol. B 24, 1365 (2006).

${ }^{2}$ P. A. Stolk, H. J. Gossman, and D. J. Eaglesham, J. Appl. Phys. 81, 6031 (1997).

${ }^{3}$ N. E. B. Cowern, K. T. F. Janssen, G. F. A. van de Walle, and D. J. Gravesteijn, Phys. Rev. Lett. 65, 2434 (1990).

${ }^{4}$ N. E. B. Cowern, G. F. A. van de Walle, D. J. Gravesteijn, and C. J. Vriezema, Phys. Rev. Lett. 67, 212 (1991).

${ }^{5}$ H. Rücker, B. Heinemann, W. Ropke, R. Kurps, D. Kruger, G. Lippert, and H. J. Osten, Appl. Phys. Lett. 73, 1682 (1998).

${ }^{6}$ S. Mirabella et al., Phys. Rev. B 65, 045209 (2002).

${ }^{7}$ W. Skorupa and R. A. Yankov, Mater. Chem. Phys. 44, 101 (1996)

${ }^{8}$ J. Tersoff, Phys. Rev. Lett. 64, 1757 (1990).

${ }^{9}$ T. R. Waite, Phys. Rev. 107, 463 (1957).

${ }^{10}$ G. Hobler and G. Kresse, Mater. Sci. Eng., B 124-125, 368 (2005).

${ }^{11}$ D. J. Bacon, D. M. Barnett, and R. O. Scattergood, Prog. Mater. Sci. 23, 51 (1979).

${ }^{12}$ R. Shneck, R. Alter, A. Brokman, and M. P. Dariel, Philos. Mag. A 65, 797 (1992).
${ }^{13}$ R. A. Masumura and G. Sines, J. Appl. Phys. 41, 3930 (1970).

${ }^{14}$ R. Bullough, M. J. Norgett, and S. Webb, J. Phys. F: Met. Phys. 1, 345 (1971).

${ }^{15}$ R. Bullough and J. R. Hardy, Philos. Mag. 17, 833 (1968).

${ }^{16}$ J. R. Hardy and R. Bullough, Philos. Mag. 15, 237 (1967).

${ }^{17}$ B. K. D. Gairola, Phys. Status Solidi B 85, 577 (1978).

${ }^{18}$ S. W. Ellaway and D. A. Faux, J. Appl. Phys., 92, 3027 (2002).

${ }^{19}$ D. P. Williams, A. D. Andreev, E. P. O'Reilly, and D. A. Faux, Phys. Rev. B 72, 235318 (2005).

${ }^{20}$ D. A. Faux and G. S. Pearson, Phys. Rev. B 62, R4798 (2000).

${ }^{21}$ D. A. Faux and U. M. E. Christmas, J. Appl. Phys. 98, 073522 (2005).

${ }^{22}$ T. Mura and N. Kinoshita, Phys. Status Solidi B 47, 607 (1971).

${ }^{23}$ E. Pan and F. Tonon, Int. J. Solids Struct. 37, 943 (2000).

${ }^{24}$ E. Pan, J. Appl. Phys. 91, 3785 (2002).

${ }^{25}$ Zi Jian, Zhang Kaiming, and Xie Xide, Phys. Rev. B 41, 12915 (1990).

${ }^{26}$ F. H. Stillinger and T. A. Weber, Phys. Rev. B 31, 5262 (1985).

${ }^{27}$ W. Smith and T. R. Forester, J. Mol. Graphics 14, 136 (1996).

${ }^{28}$ H. E. Schaefer and H. Kronmüller, Phys. Status Solidi B 67, 63 (1975). 